\title{
THE SPINOR GENUS OF QUATERNION ORDERS $\left({ }^{1}\right)$
}

\author{
BY
}

\author{
GORDON L. NIPP
}

\begin{abstract}
Let $D$ be a global domain whose quotient field $F$ does not have characteristic 2 , let $\mathfrak{U}$ be a quaternion algebra over $F$, and let D) be an order on $थ$ over $D$. $A$ right $D$-module $M$ which is simultaneously a lattice on $\mathscr{U}$ over $D$ is said to be right $\mathscr{D - g e n e r i c}$ if there exists $a \in \mathbb{U}$, $N(\alpha) \neq 0$, such that $a^{-1} M \in \operatorname{gen} \emptyset$. Our main result is that every right $\emptyset$ generic module is cyclic if and only if every class in the spinor genus of D represents a unit in $D$. One consequence is that is in a spinor genus of one class if and only if $D$-generic modules are cyclic and $D$ represents every unit represented by its spinor genus. In addition, it is shown that a necessary and sufficient condition that an integral ternary lattice $L$ be in a spinor genus of one class is that every right $\Omega_{L}$-generic pair be equivalent to a two-sided $D_{L}$-generic pair, where $\theta_{L}$ is the quaternion order associated with $L$.
\end{abstract}

1. Introduction. The notation and terminology will be that of O'Meara [5]. We will assume throughout that $D$ is a Dedekind domain whose quotient field $F$ is a global field with characteristic not 2 , and if $F$ is a rational function field, we assume $x \in D$. In addition, we assume that $\mathcal{A}$ is a quaternion algebra over $F$ and that $\mathscr{D}$ is an order over $D$ on $\mathscr{U}$ (i.e., $D$ is a lattice on $\mathcal{U}$ which is also a ring containing the identity of $\mathscr{U}$ ).

It is natural to examine the structure of the ideals of $D$. In this paper, we consider $\mathcal{D}$-modules in $\mathscr{U}$ which are simultaneously lattices on $\mathcal{U}$ over $D$, and we investigate the problem of determining when such $D$-modules are cyclic. Certainly if $M$ is such a right $\supseteqq$-module and if $M=a \supseteqq$, then $\alpha^{-1} M$ is in the genus of $\supseteqq$. Pairs $[M, a]$ satisfying this condition are called right D-generic pairs, continuing an idea of Kaplansky [3], and $M$ is said to be a right -generic module. I-generic modules are studied in $\$ 2$, and in Theorems 2.4 and 4.1 we show that a necessary and sufficient condition that right D-generic modules be cyclic is that every class in the spinor genus of $D$ represent a unit in $D$.

Received by the editors June 27, 1974.

AMS (MOS) subject classifications (1970). Primary 10C05.

(1) Most of the material in this article is from a $\mathrm{Ph} . \mathrm{D}$. dissertation written at the University of Southern California under Professor Dennis Estes. The author wishes to gratefully acknowledge Professor Estes' advice and encouragement. 
The problem of determining when two lattices in the same spinor genus are in the same class is recognized to be a difficult one. It follows as a corollary of the above theorems that a quaternion order $D$ is in a spinor genus of one class if and only if right $\mathcal{P}$-generic modules are cyclic and $\mathscr{P}$ represents every unit represented by its spinor genus. We may obtain corresponding results for ternary lattices by using the mapping $L \mapsto \bigcap_{L}$ from integral ternary lattices to quaternion orders defined by Pall [6] and generalized in [4]. Two D-generic pairs $[M, \alpha]$ and $\left[M_{1}, \alpha_{1}\right]$ are equivalent if $\alpha^{-1} M$ and $\alpha_{1}^{-1} M_{1}$ are in the same class. It is shown that an integral ternary lattice $L$ is in a spinor genus of one class if and only if every right $\vartheta_{L^{-}}$ generic pair is equivalent to a two-sided $\mathfrak{D}_{L}$-generic pair. These and other corollaries of the main theorem are incorporated into $\$ 5$.

In $\$ 6$ we give an example in which our results are used to show that the ternary lattice $L$ over the rational integers associated with the quadratic form $f\left(x_{1}, x_{2}, x_{3}\right)=x_{1}^{2}+3 x_{2}^{2}+3 x_{2} x_{3}+3 x_{3}^{2}$ and its corresponding quaternion order $\vartheta_{L}$ are each in a spinor genus of one class (although it is known that each genus contains at least two classes).

2. Igeneric modules. Let $M$ be a right (left) $\mathcal{D}$-module in $\mathscr{U}$ which is also a lattice on $\mathscr{U}$ over $D$.

Definition. $M$ is right (left) $\supseteqq$-generic if there exists $\alpha \in \mathfrak{U}, N(\alpha) \neq 0$, such that $\alpha^{-1} M \in$ gen $\supseteqq\left(M^{-1} \alpha \in\right.$ gen $\left.\supseteqq\right)$.

For purposes of precision it will sometimes be useful to specify both the $\mathcal{D}$-generic module $M$ and the corresponding element $a \in \mathscr{U}$. In such cases, we will refer to the pair $[M, a]$ as an $\supseteqq$-generic pair. We will prove our results for right $D$-generic pairs, but they hold for left $\supseteq$-generic pairs as well. If no confusion can exist, we may simply refer to a pair as being गgeneric.

According to O'Meara's definition of genus, two lattices are in the same genus only if they lie in the same quadratic space. As is seen in the next proposition, I-generic modules may be related to an extension of the concept of genus to include lattices in possibly different quadratic spaces.

Proposition 2.1. $M$ is right D-generic if and only if tinere exists $a \in \dot{F}$ such that the scaled lattice $M^{a}$ satisfies the following:

(i) at each discrete spot $p$ on $F$, there exists an isometry $\sigma_{p}: \mathfrak{O I}_{p}^{a} \rightarrow$ $\mathscr{O}_{p}$ such that $\sigma_{p} M_{p}^{a}=D_{p}$; and

(ii) at each archimedean spot $p$ on $F, \mathfrak{Y I}_{p}^{a} \simeq \mathfrak{P I}_{p}$.

Proof. If $[M, \alpha]$ is right $\mathscr{D}$-generic, let $a=N(\alpha)^{-1}$. At each spot $p$, 
the map $\sigma_{p}: \mathscr{P U}_{p}^{a} \rightarrow \mathscr{N}_{p}$ defined by $\sigma_{p}(x)=a^{-1} x$ is an isometry. If $p$ is discrete, $\sigma_{p} M_{p}^{a}=a^{-1} M_{p} \simeq \vartheta_{p}$. Hence, conditions (i) and (ii) are satisfied.

Conversely, if $M$ satisfies condition (i), then, since $D_{p}$ represents 1 ,

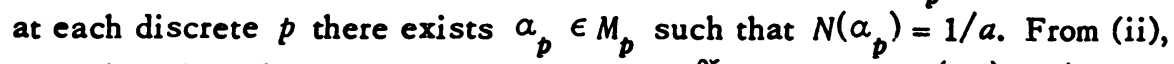
at each archimedean spot there exists $a_{p} \in \mathscr{U}_{p}$ such that $N\left(a_{p}\right)=1 / a$. By the Hasse-Minkowski Theorem, $\mathscr{U}$ represents $1 / a$. Choose $a \in \mathfrak{U}$ such that $N(\alpha)=1 / a$. Clearly, $\alpha^{-1} \cdot M$ is isometric to $M^{a}$ and $\alpha^{-1} \cdot M \in$ gen $\supseteqq$.

All spots to which we refer in the remainder of this paper will be discrete.

Definition. $\Delta(D)=16 \nu(D)$ where $\nu(\supseteqq)$ is the volume of $D$.

Since $v(\supseteqq) \subset s(\supseteqq)^{4}$, we have $\Delta(D) \subset[2 s(\supseteqq)]^{4} \subset N(\supseteqq)^{4}=D$. So $\Delta(D)$ is an integral ideal.

Definition. $M$ is right-invertible for $D$ if there exists a left $D$-module $M^{\prime}$ in $\mathscr{O}$ which is also a lattice on $\mathcal{O}$ over $D$ such that $M^{\prime} \cdot M=\bigoplus$.

It is well known that $M$ is right-invertible for $\supseteqq$ if and only if $M_{p}$ is a cyclic right $\vartheta_{p}$-module at each discrete $p$.

Theorem 2.2. $[M, a]$ is right D-generic if and only if

(i) $M$ is right-invertible for $D$,

(ii) there exists $a \in \dot{F}$ such that $N(M)=a D$,

(iii) there exists $a \in \mathfrak{U}$ such that $N(a)=a u$, where $u$ is a unit in $D$, and

(iv) at each spot $p \mid \Delta(\vartheta)$, there exists $a_{p} \in M_{p}$ such that $N\left(a_{p}\right)=a u$.

Proof. Assume $[M, \alpha]$ is right $\mathfrak{D}$-generic and let $R=\{x \in \mathscr{U}: M x \subset M\}$ be the right order of $M$. Since $\alpha^{-1} M \in$ gen $\supseteqq$, it follows from the Noether-Skolem Theorem that at each spot $p$ there exist $\delta_{p}, \gamma_{p} \in \mathfrak{O}_{p}$ such that $\alpha^{-1} M_{p}=$ $\delta_{p} \vartheta_{p} \gamma_{p}$ and $N\left(\delta_{p}\right)=N\left(\gamma_{p}\right)^{-1}$. It is easily seen that $R_{p}=\gamma_{p}^{-1 \vartheta_{p}} \gamma_{p}, v\left(R_{p}\right)=$ $v\left(\vartheta_{p}\right)$, and hence that $\bigoplus_{p}=R_{p}$ at each $p$. Thus, $\supseteqq=R$, and $\alpha^{-1} M_{p}=\delta_{p} \gamma_{p} \vartheta_{p}$ at each $p$. Setting $a=N(\alpha), u=1$, and $\alpha_{p}=a \delta_{p} \gamma_{p}$ at each $p$, conditions (iii) and (iv) are satisfied. Also since $M_{p}=a_{p} \vartheta_{p}$ at each $p, M$ is rightinvertible for $D$ and $N(M)=a D$.

Conversely, let $L=a^{-1} \cdot M$. Since $M$ is invertible, there exists $\beta_{p} \epsilon$ $M_{p}$ at each $p$ such that $M_{p}=\beta_{p} \vartheta_{p}$. It follows from (ii) and (iii) that $N\left(\alpha^{-1} \beta_{p}\right)$ is a unit in $D_{p}$; hence $\Delta(\supseteqq)=\Delta(L)$. For $p+\Delta(\supseteqq), D_{p}=\Delta\left(\vartheta_{p}\right)=$ $\Delta\left(L_{p}\right)=N\left(\bigcap_{p}\right)=N\left(L_{p}\right)$; so $\bigoplus_{p}$ and $L_{p}$ are $D_{p}$-maximal [5, 82:19]. Thus, $L_{p} \in \operatorname{cls} \vartheta_{p}$ for each $p \nmid \Delta(\supseteqq)[5,91: 2]$. Now suppose $p \Delta(\supseteqq)$. Since $M_{p}$ is invertible, it follows that $\bar{M}_{p} \cdot M_{p}=a \bigoplus_{p}$. So $\bar{\alpha}_{p} M_{p} \subset a \vartheta_{p}=\bar{\alpha}_{p} \alpha_{p} \vartheta_{p}$ and $M_{p} \subset$ $a_{p} \vartheta_{p}$. The opposite containment is clear; thus $M_{p}=a_{p} \vartheta_{p}$. Let $\xi_{p}=a^{-1_{a}} a_{p}$ The map defined by $\lambda_{p}(x)=\xi_{p} x$ is an isometry of $\vartheta_{p}$ taking $\vartheta_{p}$ onto $L_{p}$. Therefore $L_{p} \in \operatorname{cls} \bigoplus_{p}$ for each $p \mid \Delta(\supseteqq)$. Thus $L=\alpha^{-1} M \in \operatorname{gen} \supseteqq$, and $[M, a]$ is right D-generic. 
Proposition 2.3. If $[M, a]$ is right D-generic and $T$ is the left order of $M$, then $T$ and $L=\alpha^{-1} M$ are in spn $\cap$ and $[M, \alpha]$ is left $T$-generic.

Proof. As in the proof of the preceding proposition, there exist $a_{p} \epsilon$ $M_{p}$ at each $p$ such that $M_{p}=a_{p} \supseteqq_{p}$ and $N\left(a_{p}\right)=N(a)$. Thus, if $L=\alpha^{-1} M$, $L_{p}=a^{-1} M_{p}=a^{-1} \alpha_{p} \vartheta_{p}=\xi_{p} \vartheta_{p}$ where $\xi_{p}=a^{-1} a_{p}$ and $N\left(\xi_{p}\right)=1$. It follows that the spinor norm of the rotation $x \mapsto \xi_{p} x$ is one and that $L \in \operatorname{spn} \supseteqq$.

If $T=\{x \in \mathscr{U}: x M \subset M\}$, then $\alpha^{-1} T a$ is the left order of $L$ and $\xi_{p} D_{p} \xi_{p}^{-1}$ is the left order of $L_{p}$ in $\mathscr{P}_{p}$. Thus at each $p, T_{p}=a \xi_{p} \bigoplus_{p} \xi_{p}^{-1} \alpha_{p}^{-1}$, and if $\Sigma_{p}(x)=\xi_{p} x \xi_{p}^{-1}$ and $\sigma(x)=a x \alpha^{-1}$, then $\Sigma_{p} \in O^{\prime}\left(\mathscr{U}_{p}\right), \sigma \in O^{+}(\mathcal{U})$, and $T_{p}=$ $\sigma_{p} \Sigma_{p} \vartheta_{p}$ at each $p$. Therefore $T \in \operatorname{spn} \supseteqq$.

That $[M, a]$ is left $T$-generic follows from the fact that $M_{p}=T_{p} a_{p}$ at each spot and from the previous proposition.

Theorem 2.4. If every class in the spinor genus of D represents a unit in $D$, then every $\supseteqq$-generic module $M$ is cyclic.

Proof. Let $[M, a]$ be an $\mathcal{D}$-generic pair. Then $\alpha^{-1} M \in$ spn $D$. Choose $\beta \in M$ such that $N\left(\alpha^{-1} \beta\right)=u$, a unit in $D$. Thus,

$$
N(\alpha) \supseteqq=N(\beta) \mathscr{D}=\bar{\beta} \beta \mathcal{S} \subset \bar{\beta} \cdot M \subset \bar{M} M=N(\alpha) \supseteqq
$$

and $\bar{\beta} M=N(\alpha)$ D. Therefore, $M=N(\alpha) N(\beta)^{-1} \beta \cdot D=\beta$ D .

3. Preliminary results. We shall prove the converse of Theorem 2.4. To this end, it is useful to record the results which follow.

Proposition 3.1. Let $L$ and $K$ be lattices on $\mathfrak{A}$, and let $A$ be an integral ideal in $D$. Suppose that for each $p \mid A, L_{p}=K_{p}$. Then there exists $t \in D$ such that $t K \subset L$ and $|t|_{p}=1$ for each $p \mid A$.

Proof. Let $B=\{t \in D: t K \subset L\}$. If $A+B \neq D$, then $A+B \subset P$ for some proper prime ideal $P$ in $D$. But the hypotheses imply that $B$ contains an element of $D-P$ for every $P$ containing $A$. So $A+B=D$. Pick $t \in B$ and $s \in A$ such that $t+s=1$. If $\left.p|A| s\right|_{p}<$,1 ; so $|t|_{p}=1$ for each $p \mid A$.

The next proposition follows directly from Theorem 3.8 of [1], and we state it without proof.

Proposition 3.2. If $\bigoplus_{p}$ is a $D_{p}$-maximal order in $\mathscr{U}_{p}$, then either $\bigoplus_{p}$ does not represent zero nontrivially or $\vartheta_{p}$ is isomorphic to $D_{p, 2}$, the ring of $2 \times 2$ matrices over $D_{p}$.

Let \|\|$_{p}$ be the norm on $\mathcal{A}$ with respect to a fixed basis.

Lemma 3.3. Let $\beta \neq 0$ be an element of $D_{p}$. Then for $a \in \mathscr{N}_{p}$ with 
$\|a-\beta\|_{p}$ sufficiently small, $\alpha \in \mathfrak{D}_{p}$.

Proof. Let $y_{1}, y_{2}, y_{3}, y_{4}$ be a basis for $\mathscr{U}$ over $F$ and for $\mathfrak{D}_{p}$ over $D_{p}$, and set $\beta=\sum_{i=1}^{4} r_{i} y_{i}$ with $r_{i} \in D_{p}$. If $\alpha \in \mathscr{U}_{p}, \alpha=\sum_{i=1}^{4} s_{i} y_{i}$ with $s_{i} \epsilon$ $F_{p}$. If \|\|$_{p}$ denotes the norm in the basis $y_{1}, y_{2}, y_{3}, y_{4}$, then there exists a positive constant $c$ such that $c\|\gamma\|_{p}^{\prime} \leq\|\gamma\|_{p}$ for any $\gamma \in \mathscr{U}_{p}[5,101: 1]$. Suppose a satisfies

$$
\|\alpha-\beta\|_{p}<c \cdot \min _{i}\left\{\left|r_{i}\right|_{p}: r_{i} \neq 0\right\} .
$$

Then $\max \left|r_{i}-s_{i}\right|_{p}<\min _{i}\left\{\left|r_{i}\right|_{p}: r_{i} \neq 0\right\} \leq 1$. If $r_{i} \neq 0$, then $\left|r_{i}-s_{i}\right|_{p}<\left|r_{i}\right|_{p}$, and by the Principle of Domination [5, 11:2], $\left|s_{i}\right|_{p}=\left|r_{i}\right|_{p} \leq 1$. If $r_{i}=0$, $\left|s_{i}\right|_{p}=\left|r_{i}-s_{i}\right|_{p} \leq 1$. So $s_{i} \in D_{p}$ for $i=1,2,3,4$, and $a \in \mathscr{D}_{p}$.

Proposition 3.4. Let $R$ be the right order of a lattice $L \in \operatorname{spn} \supseteqq$. Then $[L, 1]$ is right $R$-generic, $L \in \operatorname{spn} R$, and $R \in \operatorname{spn} \supseteqq$.

Proof. Since $L \in$ gen $\vartheta$, at each spot $p$ there exist $\alpha_{p}$ and $\beta_{p}$ in $\mathcal{U}_{p}$ such that $N\left(a_{p}\right)=N\left(\beta_{p}\right)^{-1}$ and $L_{p}=a_{p} \cdot \vartheta_{p} \cdot \beta_{p}$. Then $R_{p}=\beta_{p}^{-1} \bigcap_{p} \beta_{p}$ and $L_{p}=\xi_{p} R_{p}$ where $\xi_{p}=\alpha_{p} \beta_{p}$. By Theorem $2.2,[L, 1]$ is right $R$-generic, and by Proposition 2.3, $L \in \operatorname{spn} R$. Hence $R \in \operatorname{spn} \supseteqq$.

Proposition 3.5. Let $L \in \operatorname{spn} \vartheta$ and let $R$ be the right order of $L$. If $R \in \mathrm{cls} \supseteqq$, and if every right $\supseteqq$-generic module is cyclic, then $L$ represents a unit in $D$.

Proof. We can write $\mathscr{\varrho}=\rho R \eta$ for some $\rho, \eta \in \mathscr{U}$, and we can find a unit $\tau \in R$ such that $1=\rho \cdot \pi \cdot \eta$. Consider the lattice $L \eta \cdot \vartheta=\rho \cdot r R \cdot \eta=\eta^{-1} R \eta$ is the right order of $L \eta$; so $L \eta$ is a right -module. Clearly $\eta^{-1} \cdot L \eta \in$ gen $\supseteqq$. Therefore $[L \eta, \eta]$ is right $\mathfrak{D}$-generic, and by assumption there exists $\xi \in \mathfrak{U}$ such that $L \eta=\xi$. So $L=\xi \oslash^{-1} \eta, D=N(L)=N\left(\xi \eta^{-1}\right) D$, and $N\left(\xi \eta^{-1}\right)$ is a unit in $D$. Clearly $\xi \eta^{-1} \in L$.

4. Cyclic modules. We now prove the converse of Theorem 2.4.

Theorem 4.1. If every 9 -generic module is cyclic, then every class in the spinor genus of $\supseteqq$ represents a unit in $D$.

Proof. Let $L$ be any lattice in the spinor genus of $\supseteqq$, and let $R$ be the right order of $L$. We shall prove the theorem by constructing a right D-generic pair whose left order is simultaneously in cls $\supseteqq$ and cls $R$ (implying $R \epsilon$ cls D). The theorem will then follow from Proposition 3.5. The pioof will be given in several steps.

(i) By Proposition 3.4, $R \in \operatorname{spn} \supseteqq$. It follows that there exist $\eta \in \mathfrak{A}$ 
and $\alpha_{p} \in \mathscr{Y}_{p}$ at each $p$ such that $\eta R_{p} \eta^{-1}=\alpha_{p} \mathfrak{\Im}_{p} \alpha_{p}^{-1}$ and $N\left(\alpha_{p}\right)$ is a square in $\dot{F}_{p}$. We will show that there exists an element $a \in \mathcal{U}$ such that at each $p \mid 2 \Delta(\Im), N(\alpha)$ is a square in $\dot{F}_{p}$ and $\alpha \Im_{p} \alpha^{-1}=\alpha_{p} \Im_{p} \alpha_{p}^{-1}$.

If $a_{p} \in F_{p}$ is given, one at each $p \mid 2 \Delta(\mathfrak{S})$, it follows from the density of $F$ in $F_{p}$ and from the Strong Approximation Theorem [5, 21:2] that there exists $a \in F$ such that $\left|a-a_{p}\right|_{p}$ is arbitrarily small for all $p \mid 2 \Delta(\Im)$. Therefore

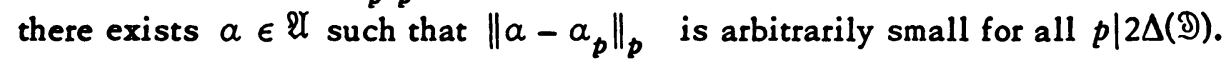
By choosing $a$ so that $\left\|\alpha-a_{p}\right\|_{p}$ is small enough, it follows [5, 101:1] that $\left\|a_{p}^{-1} a-1\right\|_{p}$ can be made arbitrarily small, and it follows from the continuity of $N$ that $\left|N(a)-N\left(a_{p}\right)\right|_{p}$ can be made arbitrarily small. By the Principle of Domination, if $\left|N(\alpha)-N\left(a_{p}\right)\right|_{p}$ is sufficiently small, then $|N(\alpha)|_{p}=$ $\left|N\left(a_{p}\right)\right|_{p}$. Also by the Local Square Theorem [5, 63:1b], if $\left|N(\alpha)-N\left(\alpha_{p}\right)\right|_{p}$ is sufficiently small, then $N(a)$ will be a square in $\dot{F}_{p}$.

Choose $a \in \mathscr{U}$ so that for every $p \mid 2 \Delta(\vartheta),\left\|a-\alpha_{p}^{\prime}\right\|_{p}$ is sufficiently small to force $a_{p}^{-1} \alpha$ to be in $\vartheta_{p}$ (using Lemma 3.3), $|N(\alpha)|_{p}=\left|N\left(a_{p}\right)\right|_{p}$, and $N(\alpha)$ to be a square in $\dot{F}_{p}$. Then since $\left|N\left(\alpha_{p}^{-1} \alpha\right)\right|_{p}=1, \alpha_{p}^{-1} \alpha$ is a unit in $D_{p}$. Thus

$$
\alpha_{p}^{-1} \alpha \cdot \mathscr{T}_{p} \cdot \alpha^{-1} \alpha_{p}=\mathscr{P}_{p} \quad \text { and } \alpha \mathscr{P}_{p} \alpha^{-1}=\alpha_{p} \mathscr{P}_{p} \alpha_{p}^{-1}=\eta R_{p} \eta^{-1} \text {. }
$$

Let $N(\alpha)=f_{p}^{2}, f_{p} \in \dot{F}_{p}$, for each $p \mid 2 \Delta(D)$.

(ii) We now show that we can assume that $N(\alpha)=a^{2} d$ where $d \in D$, $|d|_{p}=1$ at each $p \mid 2 \Delta(\supseteq)$, and $d$ is represented by a lattice in the genus of $\vartheta$.

By $[5,21: 2 a]$, at each $p \mid 2 \Delta(\supseteqq)$ there exists $\pi_{p} \in D$ such that $\left|\pi_{p}\right|_{p}$ generates $|F|_{p}=\left|F_{p}\right|_{p}$ and $\left|\pi_{p}\right|_{q}=1$ for each spot $q$ dividing $2 \Delta(\supseteq), q \neq p$. Then $f_{p}=\pi_{p}^{\circ} \epsilon_{p}$ where $o_{p}$ is an integer and $\left|\epsilon_{p}\right|_{p}=1$; so, for each $p \mid 2 \Delta(D)$, $N(a)=\pi_{p}^{2 O_{p}} \cdot \epsilon_{p}^{2}$. Set $a=\Pi_{p \mid 2 \Delta(D)} \pi_{p}^{o p}$ and $e=N(\alpha) / a^{2}$. Then $e \in F$, and for each $\left.p|2 \Delta(\mathfrak{D})| e\right|_{p}=$,1 and $e$ is the square of an element of $\dot{F}_{p}$. We observe that $e$ may be written in the form $e=b / c$ where $b$ and $c$ are in $D$ and $|b|_{p}=|c|_{p}=1$ for each $p \mid 2 \Delta(D)$. Hence $N(c a)=a^{2} \cdot b c=a^{2} d$ where $d=b c=e_{p}^{2} \in D, e_{p} \in D_{p}$, and $|d|_{p}=1$ at each $p \mid 2 \Delta(Э)$. Replacing $a$ by $c a$, we have the first part of the assertion.

Since $e_{p} \in I_{p}, d$ is represented by $\bigoplus_{p}$ for each $p \mid 2 \Delta(\Im)$. For $p \nmid 2 \Delta(\supseteqq)$, $D_{p}$ is $D_{p}$-maximal, and the set of elements represented by $D_{p}$ is the intersection of $D_{p}$ and the set represented by $\mathscr{Q}_{p}[5,91: 3]$. Since $\mathscr{U}_{p}$ is universal, $d$ is represented by $\vartheta_{p}$ for each $p$. Since $N(1 / a \cdot a)=d, d$ is represented by $\mathcal{U}$. It follows $[5,102: 5]$ that $d$ is represented by a lattice in the genus of $\supseteqq$. 
(iii) We continue by constructing an appropriate $D$-generic pair. Let $K \in$ gen $\supseteqq$ represent $d$. There exists $K^{\prime} \in$ cls $K$ such that $K_{p}^{\prime}=\bigoplus_{p}$ for each $p \mid 2 \Delta(D)[5,102: 4]$. Choose $\xi^{\prime} \in K^{\prime}$ with $N\left(\xi^{\prime}\right)=d$, and choose $t \in D$ such that $t K^{\prime} \subset \supseteq$ and $|t|_{p}=1$ at each $p \mid 2 \Delta(\vartheta)$ (using Proposition 3.1). If $\xi=$ $t \xi^{\prime}$, then $\xi$ is a unit in $\bigcap_{p}$ and $\xi^{-1 \vartheta_{p}} \xi=\bigcap_{p}$ at each $p \mid 2 \Delta(\supseteqq)$.

Let $R_{1}=\xi^{-1} \alpha^{-1} \cdot \eta R^{-1} \cdot \alpha \xi$. We note that $R_{1} \in \operatorname{cls} R$ and that for $p \mid 2 \Delta(D)$

$$
\left(R_{1}\right)_{p}=\xi^{-1} \alpha^{-1} \alpha \cdot \mathscr{P}_{p} \cdot \alpha^{-1} \alpha \xi=\xi^{-1} \cdot \mathscr{P}_{p} \cdot \xi=\mathscr{P}_{p} \cdot
$$

For $p \nmid 2 \Delta(\supseteqq),\left(R_{1}\right)_{p}=\mu_{p} \cdot \vartheta_{p} \cdot \mu_{p}^{-1}$ where $\mu_{p}=\xi^{-1} \alpha a_{p}$ and $N\left(\mu_{p}\right)$ is a square in $\dot{F}_{p}$.

Suppose $p \nmid 2 \Delta(\supseteq)$. It follows from [3, Theorem 10] that $N\left(\vartheta_{p}^{\#}\right)^{2} \Delta\left(\vartheta_{p}\right) \subset$ $16 D_{p}$ and $N\left(\vartheta_{p}^{\#}\right) \subset D_{p}$. Furthermore, since 2 is a unit in $D_{p}, \vartheta_{p} \subset \oslash_{p}^{\#}$. The maximality of $\mathscr{\vartheta}_{p}$ implies that $\mathscr{\vartheta}_{p}=\mathscr{\vartheta}_{p}^{\#}$; hence $\mathscr{\vartheta}_{p}$ is unimodular. It follows that $\vartheta_{p}$ represents zero nontrivially, and by Proposition $3.2 \vartheta_{p}$ is isomorphic to the $2 \times 2$ matrices over $D_{p}$. Therefore, for $p \nmid 2 \Delta(\vartheta)$, every two-sided ideal in $\mathscr{\vartheta}_{p}$ is of the form $E=c \vartheta_{p}$ with $c \in D$.

Now consider the right $\supseteqq$-module $R_{1} \supseteqq$. For $p \nmid 2 \Delta(\supseteqq),\left(R_{1} \supseteqq\right)_{p}=$ $\mu_{p} \mathscr{\vartheta}_{p} \mu_{p}^{-1} \mathscr{\vartheta}_{p}$. By multiplying by a suitable element of $D_{p}$, we may assume $\mu_{p}^{-1} \in \vartheta_{p}$. Then $\vartheta_{p} \mu_{p}^{-1} \vartheta_{p}$ is a two-sided ideal in $\vartheta_{p}$ and hence is of the form $c \vartheta_{p}$ for some $c \in D$. Thus, $N\left(R_{1} \supseteqq\right)_{p}=t_{p}^{2} D_{p}$ for some $t_{p} \in \dot{F}_{p}$. Noting that for $p \mid 2 \Delta(\vartheta), N\left(R_{1} \supseteqq\right)_{p}=D_{p}$, we see that $N\left(R_{1} \supseteqq\right)$ is locally the square of a fractional ideal, and we may write $N\left(R_{1} \mathscr{D}\right)=I^{2}$, where $I$ is a fractional ideal of $D$. Then $N\left(I^{-1} R_{1} \supseteqq\right)=D$, and it is a direct consequence of Theorem 2.2 that $\left[I^{-1} R_{1} \supseteqq, 1\right]$ is right $\supseteqq$-generic.

(iv) To complete the proof, we may assume by hypothesis that $I^{-1} R_{1} \supseteqq=$ $\omega \supseteqq$ for some $\omega \in \mathfrak{U}$. If $T$ is the left order of $I^{-1} R_{1} \supseteqq$, then $T=\omega \supseteqq \omega^{-1}$, $T \in$ cls $\vartheta$, and $R_{1} \subset T$. A local argument shows that $T=R_{1}$, and hence $T \epsilon$ cls $R$. Thus $R \in$ cls $\supseteqq$, and the theorem follows from Proposition 3.5.

5. Spinor genus. We have shown that if $R$ is the right order of a lattice $L \in \operatorname{spn} \supseteqq$, and if every $\supseteqq$-generic module is cyclic, then $R \in \operatorname{cls} \supseteqq$. Thus, if every 9 -generic module is cyclic, all the orders in the spinor genus of $D$ are in the same class. The following corollary is then a direct consequence of Theorem 2.4:

Corollary 5.1. If the spinor genus of a lattice $L$ contains two distinct classes each of which contains an order, then there is another class in spn $L$ which does not represent a unit. 
Corollary 5.2. $D$ is in a spinor genus composed of one class if and only if right -generic modules are cyclic and $D$ represents every unit represented by its spinor genus.

Proof. If $L$ is a lattice in spn $D$ and $R$ is its right order, then (assuming D-generic modules are cyclic) $R \in$ cls $\supseteqq$. As in the proof of Proposition 3.5 there exists $\xi \eta^{-1} \in L$ such that $N\left(\xi \eta^{-1}\right)$ is a unit and $\xi^{-1} L \eta=\vartheta$. By assumption, there exists a unit $\beta \in \mathscr{D}$ such that $N(\beta)=N\left(\xi \cdot \eta^{-1}\right)$. Thus Э $=\beta \xi^{-1} L \eta$, and since $N\left(\beta \xi^{-1}\right)=N(\eta)^{-1}, L \in$ cls $\supseteqq$.

The converse follows from Theorem 2.4 .

We remark that for the family of indefinite quaternion algebras the class and spinor genus of a lattice coincide. Thus, for any indefinite quaternion order $D$, $D$-generic modules are cyclic and $D$ represents every unit represented by its spinor genus.

Lemma 5.3. If $L \in \operatorname{spn} \supseteqq$ and $L$ is in a class which represents one, then the class of $L$ contains an order.

Proof. Choose $\mu \in L$ with $N(\mu)=1$, and let $\mathscr{D}^{\prime}=\mu^{-1} L$. $\mathscr{D}^{\prime} \in \mathrm{cls} L$, and by Proposition 3.4 and Theorem 2.2, $\bigoplus^{\prime}$ is invertible for its right order. From $[3, \S 3], D^{\prime}$ is an order.

The 9 -generic pair $[M, a]$ is said to be equivalent to the 9 -generic pair $\left[M_{1}, \alpha_{1}\right]$ if $\alpha^{-1} M$ and $\alpha_{1}^{-1} M_{1}$ are in the same class.

Proposition 5.4. There is exactly one class representing one in the spinor genus of an order $\mathfrak{D}$ in $\mathcal{U}$ if and only if every right $\mathfrak{D}$-generic pair is equivalent to a two-sided $\supseteqq$-generic pair.

Proof. From Lemma 5.3, every class in spn 9 which represents one contains an order $D^{\prime} \epsilon \operatorname{spn} D$. As in Theorem 4.1, there exist an order $R_{1} \epsilon$ cls $\mathscr{D}^{\prime}$ and a fractional ideal $I$ such that $\left[I^{-1} R_{1} \supseteqq, 1\right]$ is right $\mathscr{P}$-generic. If this pair is equivalent to the two-sided $D_{\text {generic pair }}[M, a]$, then there exist $\xi, \eta \in \mathscr{U}$ such that $\xi^{-1} R_{1} \supseteqq \eta=M$. Comparing left orders, we have $\xi R_{1} \xi^{-1}=$ D); hence $R_{1} \in$ cls $\supseteqq$ and $D^{\prime} \in$ cls $D$.

We now assume that all orders in spn $\supseteqq$ are in cls $\supseteqq$. If $[M, \alpha]$ is right D-generic, then by Proposition 2.3 the left order $T$ of $a^{-1} M$ is in spn $\mathscr{D}$ and hence in cls $\mathscr{D}$. So there exists $\eta \in \mathscr{N}$ such that $\mathscr{\vartheta}=\eta T \eta^{-1}$. Thus $\mathscr{D}$ is the left order of $\eta a^{-1} M$ and $\left[\eta \alpha^{-1} M, \eta\right]$ is left $\supseteqq$-generic. It is clearly a twosided $D$-generic pair equivalent to $[M, \alpha]$.

Let $L$ be an integral lattice on a three-dimensional quadratic space over a global field $F$ (char $F \neq 2$ ). It is possible to associate with $L$ an order 
$\vartheta_{L}$ on a quatemion algebra over $F$. This association is discussed in [4], and the results are incorporated into the next theorem.

Theorem 5.5. The integral ternary lattice $L$ is in a spinor genus of one class if and only if every right $\bigoplus_{L}$-generic pair is equivalent to a two-sided O) -generic pair.

Proof. If every right $\bigcap_{L}$-generic pair is equivalent to a two-sided $D_{L}$ generic pair, then all the orders in spn $\vartheta_{L}$ are in the same class. Using [4, 3.2 and 3.5], if $K \in \operatorname{spn} L$, then $\bigoplus_{K} \in \operatorname{spn} \bigoplus_{L}, \bigoplus_{K} \in \operatorname{cls} \bigoplus_{L}$, and $K \in \operatorname{cls} L$.

Conversely, if $\mathscr{I}$ is an order in $\operatorname{spn} \vartheta_{L}$, there is a lattice $K \in \operatorname{spn} L$ such that $\supseteqq=D_{K}[4,4.1]$. So if $L$ is in a spinor genus of one class, then all the orders in spn $\vartheta_{L}$ are in the same class. By Lemma 5.3, there is exactly one class in $\operatorname{spn} \bigoplus_{L}$ which represents one. The conclusion follows.

6. Example. Consider the free ternary lattice $L$ over the rational integers $Z$ associated with the quadratic form

$$
f\left(x_{1}, x_{2}, x_{3}\right)=x_{1}^{2}+3 x_{2}^{2}+3 x_{2} x_{3}+3 x_{3}^{2} \text {. }
$$

The corresponding quaternion order $\mathscr{D}_{L}$ (see [4]) has the associated positive definite quadratic form

$$
F\left(x_{0}, x_{1}, x_{2}, x_{3}\right)=x_{0}^{2}+3 x_{0} x_{1}+9 x_{1}^{2}+3 x_{2}^{2}-3 x_{2} x_{3}+3 x_{3}^{2}
$$

with respect to the basis $1, \alpha_{1}, \alpha_{2}, \alpha_{3}$ of $\vartheta_{L}$ corresponding to a fixed basis for $L$. Let $H$ be the symmetric matrix associated with $F$, and let $B$ and $N$ be the corresponding symmetric bilinear form and norm on $\vartheta_{L}$. We shall show that the spinor genus of $L$ and of $\bigcap_{L}$ each contains only one class.

Let $[M, \alpha]$ be a right $\mathscr{\vartheta}_{L}$-generic pair, and let $\mu_{0}, \mu_{1}, \mu_{2}, \mu_{3}$ be a basis for $M$ over $Z$. Since $N(M)=m Z$ for some positive rational number $m$ we may write $2 B\left(\mu_{i}, \mu_{j}\right)=m g_{i j}$ for $i \neq j$ and $N\left(\mu_{i}\right)=m g_{i i}$ with $g_{i j} \in Z$ for $i, j=$ $0,1,2,3$. Thus

$$
N\left(\sum_{i=0}^{3} x_{i} \mu_{i}\right)=m \sum_{i, j} g_{i j} x_{i} x_{j}=m G\left(x_{0}, x_{1}, x_{2}, x_{3}\right)
$$

with $G\left(x_{0}, x_{1}, x_{2}, x_{3}\right)$ an integral quadratic form. We use the same notation $m G$ for the matrix $\left(B\left(\mu_{i}, \mu_{j}\right)\right)$ of the basis $\mu_{0}, \mu_{1}, \mu_{2}, \mu_{3}$ of $M$.

Certainly the volumes $v\left(\alpha^{-1} M\right)$ and $v\left(\vartheta_{L}\right)$ are equal, and $N(\alpha)^{-4}[\operatorname{det}(m G)] Z=$ $(\operatorname{det} H) Z$. From Theorem 2.2, $N(\alpha)=m$, and hence $\operatorname{det} G= \pm \operatorname{det} H$. Moreover, if $T$ is the rational change of basis matrix from $\mu_{0}, \mu_{1}, \mu_{2}, \mu_{3}$ to 1 , 
$a_{1}, a_{2}, a_{3}$, then

$$
T^{\prime}(m G) T=H, \operatorname{det} G=m^{-4} \cdot(\operatorname{det} T)^{-2} \cdot \operatorname{det} H ;
$$

hence, $\operatorname{det} G=\operatorname{det} H=(27 / 4)^{2}$.

$G$ represents a positive integer $t \leq(4 \cdot \operatorname{det} G)^{1 / 4}<4[2$, p. 185]. So there exists $\xi \in M$ such that $N(\xi)=m t$ with $t=1,2$, or 3 . Since $\bar{M} M=$ $m \vartheta_{L}, \bar{\xi} M=m M^{\prime}$ where $M^{\prime}$ is a right ideal of $\vartheta_{L}$. Then $N\left(M^{\prime}\right)=t Z$ with $t=1,2$, or 3 , and $\left[M^{\prime}, \bar{\xi} \alpha / m\right]$ is right $D_{L}$-generic. We will show that $M^{\prime}$ must be cyclic.

If $\eta \in M^{\prime}$, then $\bar{\eta} \in \bigoplus_{L}, \bar{\eta} \in M^{\prime}$, and $N\left(M^{\prime}\right) \subset M^{\prime}$. So if $t=1, M^{\prime}=\bigoplus_{L}$.

We next eliminate the case $t=2$. By Theorem 2.2, $M^{\prime}$ must represent $t$ locally at each spot. We show that $M_{3}^{\prime}$, the localization of $M^{\prime}$ at the $3 Z$ adic spot on the rationals, does not represent 2. Suppose $\left(\vartheta_{L}\right)_{3}$ contains an element $y=\sum_{i=0}^{3} a_{3 i} a_{i}$ with $a_{3 i} \in Z_{3}$ satisfying $N(\gamma)=2$. We can find $a_{0}, a_{1}, a_{2}, a_{3} \in Z$ such that $\left|a_{i}-a_{3 i}\right|_{3}<1$. Then $a_{i}-a_{3 i} \in 3 Z_{3}$, and $\sum_{i=0}^{3} a_{i} a_{i}-\gamma=3 \eta$ where $\eta \in\left(\vartheta_{L}\right)_{3}$. Thus,

$$
N\left(\sum_{i=0}^{3} a_{i} a_{i}\right)=2+9 N(\eta)+3 \cdot 2 B(\gamma, \eta), \quad N\left(\sum_{i=0}^{3} a_{i} a_{i}\right)-2 \epsilon 3 Z_{3} \cap Z=3 Z,
$$

and $F\left(x_{0}, x_{1}, x_{2}, x_{3}\right) \equiv 2(\bmod 3)$ will have an integral solution. But this implies that $x_{0}^{2} \equiv 2(\bmod 3)$ which is impossible. So $\left(D_{L}\right)_{3}$ (and hence $M_{3}^{\prime}$ ) does not represent 2 . Therefore $t \neq 2$.

Now suppose $t=3$. Then $N\left(M^{\prime}\right)=3 Z$, and $3 \cdot 1,3 a_{1}, 3 a_{2}, 3 a_{3}$ are in $M^{\prime}$. The norm of $Z(3 \cdot 1)+Z\left(3 a_{1}\right)+Z\left(3 a_{2}\right)+Z\left(3 a_{3}\right)$ is $9 Z$. So $M^{\prime}$ contains an element of the form $\gamma=a_{0} \cdot 1+a_{1} a_{1}+a_{2} a_{2}+a_{3} a_{3}, a_{i} \in Z$, where not all the $a_{i}$ 's are divisible by 3 . Since 3 divides $N(\gamma)$, it follows that 3 must divide $a_{0}$. So $\beta=a_{1} a_{1}+a_{2} a_{2}+a_{3} a_{3}$ is in $M^{\prime}$. By considering the products $\beta \alpha_{2}$ and $\beta a_{3}$, it follows that $a_{3} \alpha_{1}$ and $a_{2} \alpha_{1}$ are elements of $M^{\prime}$. Since one of $a_{1}, a_{2}, a_{3}$ is not divisible by $3, a_{1} \in M^{\prime}$. Also since the norm of $Z(3 \cdot 1)+Z a_{1}+Z\left(3 a_{2}\right)+Z\left(3 a_{3}\right)$ is $9 Z, M^{\prime}$ must contain an element of the form $a_{2} a_{2}+a_{3} a_{3}$ with $a_{2}$ or $a_{3}$ (integers) not divisible by 3 . Reducing modulo 3 and multiplying by -1 if necessary, we have the following cases:

I. If $a_{2}=1$ and $a_{3}=0$, then $a_{2} \in M^{\prime}, \bar{a}_{2} M^{\prime} \subset 3 \bigcap_{L}$, and $a_{2} \bar{a}_{2} M^{\prime}=$ $3 M^{\prime} \subset 3 a_{2} \cdot \vartheta_{L}$. So $M^{\prime} \subset a_{2} \vartheta_{L} \subset M^{\prime}$, and $M^{\prime}=a_{2} \vartheta_{L}$.

II. If $a_{2}=0$ and $a_{3}=1$, then as in case I, $M^{\prime}=a_{3} D_{L}$.

III. If $a_{2}=a_{3}=1$, then $a_{2}+a_{3} \in M^{\prime}$, and since $N\left(a_{2}+a_{3}\right)=3$, we have $M^{\prime}=\left(a_{2}+a_{3}\right) \vartheta_{L}$.

IV. If $a_{2}=1$ and $a_{3}=-1$, we observe that the norm of $Z(3 \cdot 1)+$ 
$Z a_{1}+Z\left(3 \cdot a_{2}\right)+Z\left(a_{2}-a_{3}\right)$ is $9 Z$. So $M^{\prime}$ contains an element to which case I applies.

In all cases, $M^{\prime}$ is cyclic; so $M$ is cyclic. Furthermore, since $F$ is positive definite, the unit -1 is not represented. The hypotheses of Corollary 5.2 are satisfied; hence $\bigcap_{L}$ is in a spinor genus of one class. By Proposition 5.4 and Theorem 5.5, $L$ is in a spinor genus of one class.

We remark that the genus of $L$ contains at least two classes [7, p. 115]. It follows from [4] that the genus of $\bigcap_{L}$ contains an order not in the spinor genus of $\mathfrak{D}_{L}$.

\section{REFERENCES}

1. M. Auslander and O. Goldman, Maximal orders, Trans. Amer. Math. Soc. 97 (1960), 1-24. MR 22 \#8034.

2. L. E. Dickson, Studies in the theory of numbers, Chelsea, New York, 1930.

3. I. Kaplansky, Submodules of quatemion algebras, Proc. London Math. Soc. (3) 19 (1969), 219-232. MR 39 \# 1496.

4. G. Nipp, Quaternion orders associated with temary lattices, Pacific J. Math. 53 (1974).

5. O. T. O'Meara, Introduction to quadratic forms, Die Grundlehren der math. Wissenschaften, Band 117, Academic Press, New York; Springer-Verlag, Berlin, 1963. MR $27 \# 2485$.

6. G. Pall, On generalized quaternions, Trans. Amer. Math. Soc. 59 (1946), 280-332. MR 8, 318.

7. G. L. Watson, Integral quadratic forms, Cambridge Univ. Press, Cambridge, 1960.

DEPARTMENT OF MATHEMATICS, CALIFORNIA STATE UNIVERSITY, LOS ANGELES, CALIFORNIA 90032 